\title{
Study on Site Selection of the New High-Speed Rail Station
}

\author{
Gui-Yan SUN*
}

Liaoning Railway Vocational and Technical College, Jinzhou, Liaoning 121000, China

sgylnjz2006@163.com

Key words: High-Speed Railway (HSR), Station, Site.

\begin{abstract}
The site of the new high speed rail station should be based on driving the city's economic development and facilitating the travel of residents as a precondition. The designers should also take such factors as he size of the city, the economic level, the long-term planning of the city, the existing transport system into consideration. Only when the site selection of the high speed rail station is reasonable can it truly plays its role in contributing to the development of the city and the regional economy. This article discusses the problems that should be considered in the site selection of HSR stations from different perspectives and puts forward some suggestions on how to choose a suitable site.
\end{abstract}

With the rapid development of China's high speed railway, the site of the high-speed rail station has attracted more and more attention. Whether the station site is reasonable affects the convenience of passenger's travelling, and also affect economic development of the city where the station located in[1]. If the station location is reasonable, it will effectively promote the traffic, business, economy, real estate and other areas in the surrounding areas. On the contrary, if the station location is unreasonable, for example, the new site is too far away from the main urban area and the new site is far from the city Unbalanced development planning, the new site is not easy to achieve the existing seamless connection of various modes of transport, etc. This will not only affect the convenience of travelers' travelling, but also will not promote the rapid economic development around the station.

\section{Types of the New High-Speed Rail Stations}

According to the different nature of station technical operations, there are mainly three types of high-speed railway stations in our country, that is, overtaking stations, intermediate stations and initial (final) stations.

(1) Overtaking Stations: Such stations are mainly for the passage of high-speed passenger trains and medium-speed passenger trains waiting to avoid high-speed passenger trains, and generally do not handle passenger business operations.

(2) Intermediate Station: Located at the railway hub of large and medium-sized cities along the high-speed railway, it mainly handles the passage and cross-over of high-speed passenger trains. Most of the stations in China high-speed railway lines belong to this type.

(3) Origin (final) Station: Set at the beginning and end of the high-speed rail line, located in metropolitan railway hub. Mainly for high-speed passenger train departure, final and passenger business operations.

\section{The Influence of New High-Speed Rail Station to Passenger Flow in a City \\ Characteristics of the Passengers in Rail Stations in China \\ Obvious Characteristics}

Analysis of the characteristics of China's high-speed rail passenger flow, they are the followings:

(1)Tourist Passenger Flow: With the rapid development of economy and society, the continuous improvement of people's living standard, the advantages of high-speed railway in comfort, speed and convenience have become more and more obvious. There is an increasing number of people travel on weekends and holidays, and the travelling distance becomes further and further. If 
high-speed trains run faster, travelers will save a lot of time; because of its good comfort, after getting off, short-haul passengers will not feel too tired. They can go to play directly, which virtually saves travelers travelling time and fees. Therefore, many tourists give priority to travel by high-speed rails.

(2)Business Passenger Flow: Most of these passengers are of high consumption level[2]. They have strong ability to pay, and have higher requirements on the safety, comfort, convenience, punctuality and other aspects of the transportation. High-speed rail passengers, especially in the short-distance, most of the passengers belong to business passenger flow.

(3)Visiting Relatives and Students Passengers: they are working or learning in other places, where the travel distance is halfway from home. When in weekends or holidays, they could visit relatives or family members. high-speed rail has become their preferred choice of transport, for that it is comfortable, fast and the rails are also have many courses. This kind of passengers are in an increasing number year by year.

In addition, high-speed rail passenger also contains business passenger flow, commuter passenger flow and so on.

\section{High-Speed Rail Passengers Fluctuate Significantly and Regularly}

China's high-speed rail passengers fluctuate significantly and regularly[3], especially in holidays, passengers will increase obviously. Because high-speed railways have reduced people's travel time and shorten the distance between cities, a large number of holiday passengers have been added for the purpose of leisure travel or visiting relatives and friends[4]. In addition, affected by factors such as traditional folk customs and living habits in China, large numbers of personnel movements and even staged tensions occur during the Spring Festival, the Ching Ming Festival, the Labor Day, the National Day and other summer holidays.

\section{High-Speed Rail Station Site Impacts on Passenger Flow}

The site of the new high-speed rail station should fully consider the coordination among various modes of transport and adhere to the "big traffic" train of thought. In order to give full play to the advantages of high-speed railway with high speed and large transportation capacity, it is necessary to disperse the passenger arriving at the station as soon as possible, which requires "seamless convergence" among various modes of transportation within the city. That is, high-speed railway stations, existing stations, urban rail transit, bus, taxi and other modes of transport should coordinate operation, complement each other and to better passenger transport services.

Passengers at new HSR stations will not increase significantly in the initial period. However, with the gradual improvement of the urban transport system, the effective convergence of various means of transport and the establishment of supporting facilities around the station, the number of passengers will surely be achieved.

\section{The Impact of the New High-Speed Rail Stations to City's Development}

In the past few years, there have been some problems in the site election of high-speed rail stations in China. Among them, the most prominent problem is that the new HSR station is too far away from the city center, therefore, other modes of transport are not effectively linked. It is not convenient to passengers' traveling and passenger diversion is in a slow speed. In spite of such problems, in the long run, new high-speed rail stations will promote the development of the city.

\section{The New HSR Station will expand the City Hinterland}

As the high-speed rail runs fast, there will not be too many sites along the line, and some central cities tend to become high-speed rail convergence points. These cities themselves have strong economic fundamentals. The convergence of high-speed railways has further strengthened their geographical advantages with obvious enhancement of their location. For the current study, the agglomeration effects of population and economic factors in China's future central cities, especially those with high-speed railway lines, will increase sharply[5]. In order to facilitate the travelers' 
traveling, other transport systems are generally completed to achieve the link between the newly constructed HSR station and the city center, so as to gradually expand the hinterland of the city.

\section{New High-Speed Rail Station will Enhance the City's Economic Development}

New high-speed rail stations are often far from the city center. With the opening of high-speed rail lines, the gradual convenience of urban traffic and the gradual increase of passengers, the residents around the station will gradually become crowded. Real estate and commercial economy will develop and the urban economy will develop accordingly.

\section{New High-Speed Rail Station Will Promote the Development of Regional Economy}

The rapid development of high-speed rail, especially the construction of a large number of inter-city railroads, has promoted the process of urban integration along the route. It is mainly reflected in the work of living in different places, tourism, urbanization, industrial distribution, urbanization, traffic integration, education and medical public service sharing. Through the reasonable flow and effective allocation of urban resources in space, the utilization efficiency of resources has been utilized and the development of regional economy has been promoted.

\section{Suggestion on the Rationality Plan of Site Selection for High Speed Railway Station \\ Closely Integrated with Urban Planning to Build a City Integrated Transport Hub}

Of the three types of high-speed rail stations, the initial (final) and intermediate stations often require new construction and high and medium-speed trains. Therefore, the site selection for such stations is very important.

(1) Initial (final) station:Such stations are located in the large and medium-sized railway hubs along the high-speed railways, with large passenger traffic and some provincial capitals. Station location should be closely around the city's long-term planning program, and should be located within the scope of development of the city. This site selection not only facilitates passengers' travelling, expands the size of the city, relieves traffic pressure and stimulates local economy, but even plays a positive role in alleviating traffic, employment and housing pressure in first-tier cities in the long run.

In order to facilitate the transfer of passengers, on the one hand, the designers should pay attention to mutual communication with the rail transit and realize the convenient transfer between rail transit and urban rail transit; on the other hand, it is also possible to construct a railway tie line to link up with the existing railway main and branch lines. In this way, a seamless transition between vehicles could be realized.

(2) Intermediate station:The intermediate station occupies a larger proportion in China's passenger dedicated transport line. Such stations are often located in the center of the local administrative region, the population is relatively large, and has a considerable passenger flow. However, cities with relatively small sizes and relatively small population will not be able to build transportation facilities such as subways and light rails in a short term. Therefore, passengers can only choose public transport, taxis and private cars for their trips. If the location of the station is too far from the city, it will increase the actual transit time of passengers and directly cause a loss of some passengers. Therefore, the intermediate station, as far as possible, in the city's main urban and long-term planning of urban new district on the site connection, and should not be too far from the main city. In this way, it will be both convenient to passengers' travelling, but also save the urban construction investment. In the meantime, it enable to the transition, the real pull the suburbs and the overall development of the new city.

\section{Investigation, Study, and Listen to the Opinions of the Public}

The construction of a high-speed railway is a long-term project benefiting the country and its people. The ultimate goal is to provide travel services for passengers. People who travel a lot have the right to determine how to elect high-speed rail station sites in order to coordinate with the city's long-term planning and could facilitate passenger travel. Thus, in the process of site selection, the 
designers had better to listen to the suggestions of local governments. Considering the city's long-term planning independently is not a good choice. It is necessary to conduct surveys among stations, universities and colleges, large and medium-sized enterprises and government agencies on the basis of referring to the urban planning plan and widely listen to opinions of the masses. Users can also choose the best answer from the alternatives through a network platform. If necessary, hearings can be held to make the site selection of high-speed rail more reasonable.

\section{If Stations will be built in the Tourism-Based Cities, the Site should not be too Far Away from} the Scenic Areas.

In China, the tourism resources are very abundant, and the tourism revenue accounts for a large proportion of the gross national income. Therefore, it is reasonable to build stations in some famous cities with beautiful scenery along the high-speed rail line. When setting up a station, designers should consider the convenience to the tourists firstly, thus, the station should not be too far away from the scenic area. In addition, due to seasonal tourism, the opening hours of high-speed rails can be adjusted appropriately with the changing seasons.

\section{Conclusion}

To sum up, the site of high-speed rail station should be driven by the city's economic development[6], and facilitate the travel of residents as a prerequisite. Designers must give full consideration to such factors as the size of the city, the economic level, the long-term planning of the city, the existing transport system. Only when the site selection is reasonable can it truly plays its role in contributing to the development of cities and regional economies.

\section{References}

[1]Han Yao,ZeLiu,Xiao-Chen Liu.Spatial Characteristics, Influencing Mechanism and Typical Model of Urban Spatial Development Along High Speed Rail: A Case Study of Beijing-Shanghai Express Railway.Huazhong Architecture,2015(5):7-13. (In Chinese)

[2] Cui Zhang.Analysis on Passenger Flow Characteristics of High-speed Railway Passenger

Stations in Cities of Different Grades.Railway Transport and Economy,2014(9):63-67. (In Chinese)

[3]Xue-Bin Jiang.Thoughts on Passenger Flow Principle and Marketing Strategies of Beijing-Shanghai High-speed Railway.Railway Transport and Economy,2014(2):48-51.(In Chinese)

[4]Zhen-LiZHANG. Adapt to the Passenger Flow Characteristics of China's High Speed Rails [J], Railway Transport and Economy, 2010(6):42-45. (In Chinese)

[5]Bo, JIANG, Nan-Chen CHU, Yuan WANG, Yu-Yuan MA, Xue-Song ZHANG. Review and Prospect of Researches on the Impact of High Speed Rails on Urban and Regional Space [J], Human Geography, 2016(1):16-25. (In Chinese)

[6]Lan Wang.Research Framework of High-Speed Railway Impact on Urban Space.Planners,2011(7):13-19. (In Chinese) 